\title{
Anonymity-Proof Voting Rules
}

\author{
Vincent Conitzer \\ Duke University \\ Department of Computer Science \& \\ Department of Economics \\ conitzer@cs.duke.edu
}

\begin{abstract}
A (randomized, anonymous) voting rule maps any multiset of total orders of (aka. votes over) a fixed set of alternatives to a probability distribution over these alternatives. A voting rule $f$ is neutral if it treats all alternatives symmetrically. It satisfies participation if no voter ever benefits from not casting her vote. It is falsename-proof if no voter ever benefits from casting additional (potentially different) votes. It is anonymity-proof if it satisfies participation and it is false-name-proof. We show that the class of anonymity-proof neutral voting rules consists exactly of the rules of the following form. With some probability $k_{f} \in[0,1]$, the rule chooses an alternative at random. With probability $1-k_{f}$, the rule first draws a pair of alternatives at random. If every vote prefers the same alternative between the two (and there is at least one vote), then the rule chooses that alternative. Otherwise, the rule flips a fair coin to decide between the two alternatives.
\end{abstract}

\section{Introduction}

In many settings, a decision must be made on the basis of the preferences of multiple parties (typically referred to as agents in economics). Common examples include auctions and exchanges (where we must decide on an allocation of resources, as well as payments to be made or received by the agents) and elections (where we must decide on one or more political representatives), but there are many other applications. A (directrevelation) mechanism takes each agent's reported preferences as input, and produces a decision as output. An important issue is that self-interested agents will lie about their preferences if they perceive it to be to their advantage to do so. Mechanism design (sometimes viewed as a subfield of game theory) studies how to design mechanisms that produce good outcomes in spite of this. A key concept in mechanism design is that of strategy-proofness: a mechanism is strategy-proof if no agent can ever benefit from lying about her preferences. Strategy-proofness is roughly synonymous with truthfulness and incentive compatibility. ${ }^{1}$ In mechanism design, attention is usually restricted to incentive compatible direct-revelation mechanisms. This is justified by a result known as the revelation principle [29, 31, 43, 44], which states (roughly) that, given that agents will misreport their preferences if they perceive this to be to their benefit, anything that

\footnotetext{
${ }^{1}$ To be more precise, strategy-proofness as the term is used here corresponds to dominant-strategies incentive compatibility. There are weaker notions of incentive compatibility, such as Bayes-Nash incentive compatibility, where in expectation over the other agents' preferences an agent is best off reporting her true preferences.
}

Dagstuhl Seminar Proceedings 07271 
can be achieved by some mechanism can also be achieved by an incentive compatible direct-revelation mechanism. ${ }^{2}$

In recent years, there has been an explosion of interest in mechanism design in the theoretical computer science community, as well as in the artificial intelligence community. This interest is driven in part by necessity, as computer scientists encounter more settings in which multiple self-interested agents must interact; and in part by opportunity, as there are many issues in mechanism design that are fundamentally computational in nature. Research directions include (this list is nowhere near exhaustive): algorithmic mechanism design [45], where the goal is to make algorithms in distributed settings incentive compatible; (closely related) the design of incentive compatible approximation algorithms in settings where standard mechanisms are computationally hard to execute (e.g. $[41,3,40]$ ); automated mechanism design, where the problem of finding an optimal mechanism is cast as an optimization problem (e.g. [20]); the design of online mechanisms where agents arrive and depart over time (e.g. [34, 33, 12, 4]); and applying techniques from machine learning theory to mechanism design [5]. It is also increasingly common for computer scientists to develop new characterizations of feasible mechanisms (e.g. [39, 10, 42]); while such work is not in and of itself computational in nature, it can be of great help in identifying computationally tractable mechanisms.

In mechanism design, the spaces of possible outcomes and preferences often display a great deal of structure, which facilitates the designer's job. For example, in auction settings, it is generally assumed that agents can make and receive payments, and moreover that their utility is linear in this payment. This enables, for example, Vickrey-ClarkeGroves mechanisms [54, 14, 32], which always choose the efficient allocation. However, such structure is not always available: for example, in an election, payments can typically not be made. If we do not assume any structure on the agents' preferences, then agents can rank the possible outcomes (aka. alternatives) in any possible way. These general settings, in which each agent ranks all the alternatives, and the mechanism chooses an alternative based on these rankings, are commonly referred to as voting settings. The rankings are the votes, and the mechanism is usually called a voting rule. Voting is also a topic of growing interest to computer scientists. Strategic considerations aside, some voting rules are computationally hard to execute $[8,36,15,26,52,50,13]$, leading to the use of approximation algorithms and heuristics $[26,2,1,24]$ as well as searchbased approaches $[25,18,16]$. There has also been work characterizing the communication complexity of various voting rules [21]. Additionally, researchers have investigated how computationally hard it is to find a way of misreporting one's preferences that is beneficial $[7,6,19,27,22,48,49,35,23,50]$, as well as whether there are computational barriers to other undesirable behavior, for example by the chairperson of the election $[9,37,28,50]$. Finally, there has been work on concisely representing preferences in voting $[38,55]$, as well as on extending voting theory to allow for partial preferences [46, 51, 47].

The revelation principle applies to voting settings just as it does to any other mechanism design setting, so we should ask which rules are strategy-proof. Gibbard [30] provides a complete characterization of strategy-proof voting rules that are allowed to use randomization. (This characterization generalizes the better-known, earlier GibbardSatterthwaite theorem $[29,53]$.) He shows that any strategy-proof rule is a randomization over unilateral rules, in which only one vote affects the outcome, and duple rules, in

\footnotetext{
${ }^{2}$ To predict what will happen under a mechanism that is not incentive compatible, some solution concept from game theory must be used, and the version of incentive compatibility in the revelation principle depends on the choice of solution concept.
} 
which only two alternatives have a chance of winning. (Because the overall rule is a randomization over such rules, it can still be the case that every voter affects the probability with which an alternative is chosen, and that every alternative has a positive probability of winning.) He also provides some additional conditions on these rules to obtain an exact characterization of the strategy-proof voting rules.

However, especially from the perspective of a computer scientist, strategy-proofness is often not sufficient. In open, anonymous environments such as the Internet, an agent can manipulate the mechanism in other ways. For one, if an agent does not participate in the mechanism, then the party running the mechanism (aka. the center) is not even aware of her existence. Perhaps more significantly, an agent can open multiple accounts and participate in the mechanism multiple times under different identifiers-and the center cannot know which identifiers correspond to the same agent. This led to the concept of false-name-proofness $[58,59]$. A mechanism is false-name-proof if an agent can never benefit from using multiple identifiers. Various positive and negative results on false-name-proofness have been obtained for combinatorial auctions and similar settings (e.g. $[58,56,59,57])$, but to our knowledge this concept has not yet been studied in the literature on voting.

In this paper, we define a (possibly randomized) voting rule to be anonymity-proof if it is false-name-proof, and it never hurts an agent to cast her (true) vote. Under the same model as Gibbard [30], we obtain a complete characterization of the anonymity-proof neutral voting rules. (A voting rule is neutral if it treats all alternatives symmetrically.) The proof is from first principles and (arguably) of reasonable length. The resulting class of voting rules is very limited (hence the result is mostly negative), but it does allow a modicum of responsiveness to the votes in cases where there is complete agreement among the voters on some pairs of alternatives. For example, in the special case where there are only two alternatives, the characterization tells us that if all votes prefer the same alternative, we can choose that alternative; but otherwise, we have to flip a fair coin to decide between them. This is in stark contrast to the case where we require only strategyproofness: simply choosing the alternative that is preferred by more voters (the majority rule) is strategy-proof.

\section{Definitions}

Let $X,|X|=m$, be the set of alternatives over which the voters are voting. A voter's preferences are given by a total order $\succ$ over the alternatives, together with a vector of utilities $\vec{u}=\left(u_{1}, \ldots, u_{m}\right)$ where $u_{i}$ is the voter's utility for the alternative that she ranks $i$ th. (It is required that $u_{i}>u_{i+1}$ for all $1 \leq i \leq m$.) Each voter seeks to maximize her expected utility. As in Gibbard [30], voters only report a total order (ranking) of the alternatives (not their utilities); a reported ranking is called a vote. Again as in Gibbard [30], we do not allow for indifferences (real or reported) between alternatives. We will use the notation $v=a_{1} \succ \ldots \succ a_{m}$ for a vote. We will sometimes also use subsets in the order notation: for example, if $B=\left\{b_{1}, b_{2}, b_{3}\right\}$, then $a_{1} \succ b_{1} \succ b_{2} \succ b_{3} \succ a_{2}$ and $a_{1} \succ b_{3} \succ b_{1} \succ b_{2} \succ a_{2}$ are both of the form $a_{1} \succ B \succ a_{2}$ (but, for instance, $a_{1} \succ b_{3} \succ b_{2} \succ a_{2} \succ b_{1}$ is not of this form). A voting rule $f$ takes a multiset ${ }^{3}$ of votes $V$ as input, and chooses the winning alternative based on these votes (possi-

\footnotetext{
${ }^{3}$ This is implicitly assuming that every vote is treated equally; anything else would seem unreasonable in open, anonymous environments. Rules that treat every vote equally are commonly called anonymous; this is not to be confused with the definition of anonymity-proofness.
} 
bly using randomization). Let $P_{f}(V, a)$ denote the probability with which $f$ chooses $a$ given votes $V$; the function $P_{f}$ defines the rule $f$. A voting rule is neutral if it treats all alternatives symmetrically - that is, if $\pi$ is a permutation of the alternatives, then $P_{f}(\pi(V), \pi(a))=P_{f}(V, a)$ (where $\pi(V)$ is the multiset that results from replacing each alternative $a$ by $\pi(a)$ in each vote in $V$ ). In fact, the following weaker definition of neutrality will also suffice for our purposes: if a subset $B$ of the alternatives is symmetric in $V$ (that is, for any permutation $\pi$ for which $\pi(a)=a$ for all $a \in X-B, \pi(V)=V$ ), then $P_{f}\left(V, b_{1}\right)=P_{f}\left(V, b_{2}\right)$ for all $b_{1}, b_{2} \in B$. We are only interested in neutral voting rules. ${ }^{4}$

Definition $1 A$ voting rule $f$ is false-name-proof if for any multiset of votes $V$, for any $v \in V, v=a_{1} \succ \ldots \succ a_{m}$, for any decreasing $\vec{u}=\left(u_{1}, \ldots, u_{m}\right)$, and for any multiset of votes $V^{\prime}, \sum_{j=1}^{m} P_{f}\left(V, a_{j}\right) u_{j} \geq \sum_{j=1}^{m} P_{f}\left(V \cup V^{\prime}, a_{j}\right) u_{j}$. That is, the voter corresponding to $v$ cannot increase her expected utility by additionally casting votes $V^{\prime}$.

It should be noted that under this definition, a voter who uses false names is assumed to cast at least one vote representing her true preferences. This only weakens the requirement. All of the rules in the characterization result of this paper are also false-name-proof in the stronger sense where none of the votes cast by the false-name voter are required to represent her true preferences. Hence, the characterization remains the same if this stronger requirement is used.

Definition 2 A voting rule $f$ satisfies participation if for any multiset of votes $V$, for any $v \in V, v=a_{1} \succ \ldots \succ a_{m}$, for any decreasing $\vec{u}=\left(u_{1}, \ldots, u_{m}\right), \sum_{j=1}^{m} P_{f}\left(V, a_{j}\right) u_{j} \geq$ $\sum_{j=1}^{m} P_{f}\left(V-\left\{v^{\prime}\right\}, a_{j}\right) u_{j}$. That is, the voter corresponding to $v$ cannot increase her expected utility by not casting her vote.

Definition 3 A voting rule is anonymity-proof if it is false-name-proof and it satisfies participation.

Anonymity-proofness does not directly mention strategy-proofness. Thus, it may appear that even if a rule is anonymity-proof, it is possible that a voter can benefit from misreporting her preferences. However, all of the rules in the characterization result of this paper are also strategy-proof (this is implied by the fact that they satisfy the stronger version of false-name-proofness). Hence, the characterization remains the same if strategyproofness is added as a requirement.

\section{The characterization of anonymity-proof rules}

In this section, we prove the main result. The proof mostly consists of six lemmas; the main theorem follows quite easily from the last three lemmas.

The first lemma is a fundamental building block of the proof. It states that if we add a vote that agrees with an existing vote on the top $k$ and bottom $l-k$ alternatives, then the probability of winning for each of those alternatives does not change.

Lemma 1 Consider a multiset of votes $V$, and suppose that for some $v \in V$, $v$ is of the form $a_{1} \succ \ldots \succ a_{k} \succ B \succ a_{k+1} \succ \ldots \succ a_{l}$. Let $v^{\prime}$ (not necessarily in $V$ ) be another

\footnotetext{
${ }^{4}$ Sometimes rules that are not neutral are of interest, for example if one alternative is the incumbent and should be treated specially; but in most settings, only neutral rules are of interest.
} 
vote of the form $a_{1} \succ \ldots \succ a_{k} \succ B \succ a_{k+1} \succ \ldots \succ a_{l}$ (that is, it is identical to $v$ except for the internal ordering of $B$ ). Then, if $f$ is anonymity-proof, for any $1 \leq i \leq l$, $P_{f}\left(V, a_{i}\right)=P_{f}\left(V \cup\left\{v^{\prime}\right\}, a_{i}\right)$.

Proof: First, let us suppose that for some $1 \leq i \leq k, P_{f}\left(V, a_{i}\right) \neq P_{f}\left(V \cup\left\{v^{\prime}\right\}, a_{i}\right)$. Without loss of generality, suppose that for any $1 \leq j<i, P_{f}\left(V, a_{j}\right)=P_{f}\left(V \cup\left\{v^{\prime}\right\}, a_{j}\right)$. Consider the utility vector $\vec{u}=(1-\epsilon, 1-2 \epsilon, \ldots, 1-i \epsilon,(m-i) \epsilon,(m-i-1) \epsilon, \ldots, \epsilon)$. First, let us suppose that $P_{f}\left(V, a_{i}\right)<P_{f}\left(V \cup\left\{v^{\prime}\right\}, a_{i}\right)$. Then, if the true preferences are given by $V$, the voter casting $v$ has utility vector $\vec{u}$, and $\epsilon$ is sufficiently small, then the voter casting $v$ has an incentive to cast $v^{\prime}$ as well. This is because (as $\epsilon \rightarrow 0$ ) she effectively seeks to maximize the probability of one of $a_{1}, \ldots, a_{i}$ winning, and casting $v^{\prime}$ as well does not affect the probabilities of $a_{1}, \ldots, a_{i-1}$ winning and increases that of $a_{i}$. On the other hand, suppose that $P_{f}\left(V, a_{i}\right)>P_{f}\left(V \cup\left\{v^{\prime}\right\}, a_{i}\right)$. Then, if the true preferences are given by $V \cup\left\{v^{\prime}\right\}$, the voter casting $v^{\prime}$ has utility vector $\vec{u}$, and $\epsilon$ is sufficiently small, then the voter casting $v^{\prime}$ has an incentive to not participate. This is because (as $\epsilon \rightarrow 0$ ) she effectively seeks to maximize the probability of one of $a_{1}, \ldots, a_{i}$ winning, and not participating does not affect the probabilities of $a_{1}, \ldots, a_{i-1}$ winning and increases that of $a_{i}$. Hence, for any $1 \leq i \leq k, P_{f}\left(V, a_{i}\right)=P_{f}\left(V \cup\left\{v^{\prime}\right\}, a_{i}\right)$.

The case where $P_{f}\left(V, a_{i}\right) \neq P_{f}\left(V \cup\left\{v^{\prime}\right\}, a_{i}\right)$ for some $k+1 \leq i \leq l$ can be shown to contradict either false-name-proofness or participation by a symmetric argument (where, supposing without loss of generality that $P_{f}\left(V, a_{j}\right)=P_{f}\left(V \cup\left\{v^{\prime}\right\}, a_{j}\right)$ for all $i<j \leq l$, the voter casting $v$ or $v^{\prime}$ effectively tries to minimize the probability of one of the last $l-i+1$ alternatives winning).

We obtain the following corollary, which states that it does not matter if the same vote is cast more than once. (This corollary is usually not powerful enough to use instead of the more general Lemma 1, but it provides some insight.)

Corollary 1 For an anonymity-proof rule $f$, given that a vote is cast at least once, it does not matter how often it is cast.

Proof: This follows from setting $B=\emptyset$ in Lemma 1 .

Lemma 1 allows us to prove the following lemma, which states that reordering the alternatives after a given alternative $b$ in a vote, as well as reordering those before $b$, does not affect $b$ 's probability of winning, unless we move alternatives past $b$.

Lemma 2 Consider a multiset of votes $V$, and suppose that for some $v \in V$, $v$ is of the form $A \succ b \succ C$. Let $v^{\prime}$ (not necessarily in $V$ ) be another vote of the form $A \succ b \succ C$ (that is, it is identical to $v$ except for the internal ordering of $A$ and $C$ ). Then, if $f$ is anonymity-proof, $P_{f}(V, b)=P_{f}\left((V-\{v\}) \cup\left\{v^{\prime}\right\}, b\right)$. That is, we can permute the alternatives on either of $b$ in a vote without affecting $b$ 's probability of winning.

Proof: Suppose first that we permute only $C$, that is, that $A$ is ordered the same way in both $v$ and $v^{\prime}$. Then, we can apply Lemma 1 (letting $b$ correspond to $a_{k}$ in that lemma) to obtain $P_{f}(V, b)=P_{f}\left(V \cup\left\{v^{\prime}\right\}, b\right)$, and similarly $P_{f}\left((V-\{v\}) \cup\left\{v^{\prime}\right\}, b\right)=P_{f}(V \cup$ $\left.\left\{v^{\prime}\right\}, b\right)$, hence $P_{f}(V, b)=P_{f}\left((V-\{v\}) \cup\left\{v^{\prime}\right\}, b\right)$. The case where we permute only $A$ can be proven symmetrically. But then, in the general case where both $A$ and $C$ are permuted, we can transform $v$ into $v^{\prime}$ in two steps, as follows. Let $v^{\prime \prime}$ be the vote of the form $A \succ b \succ C$ that agrees with $v$ on $A$ but with $v^{\prime}$ on $C$. By the above, we have 
$P_{f}(V, b)=P_{f}\left((V-\{v\}) \cup\left\{v^{\prime \prime}\right\}, b\right)=P_{f}\left((V-\{v\}) \cup\left\{v^{\prime}\right\}, b\right)$.

The next lemma shows that we can move an alternative $b$ past a given alternative $a$ in a vote, without affecting $a$ 's probability of winning, if the other votes disagree on the relative ranking of $a$ and $b$.

Lemma 3 Consider a multiset of votes $V$, and suppose that for some $v \in V$, a is ranked before $b$. Additionally, suppose there is another vote $v^{\prime} \in V$ that ranks a before $b$, and a third vote $v^{\prime \prime} \in V$ that ranks $b$ before $a$. Let $v^{\prime \prime \prime}$ be a vote (not necessarily in $V$ ) that is obtained from $v$ by improving b's position, placing it somewhere ahead of a (while not changing the order in any other way). Then, if $f$ is anonymity-proof, $P_{f}(V, a)=$ $P_{f}\left((V-\{v\}) \cup\left\{v^{\prime \prime \prime}\right\}, a\right)$. That is, we can move b to the other side of a in a vote without affecting $a$ 's probability of winning, if there are other votes that rank $a$ before $b$ and $b$ before $a$.

Proof: Let us first assume that $a$ and $b$ are adjacent in $v$ and $v^{\prime \prime \prime}$. That is, $a$ is ranked directly before $b$ in $v$, and $v^{\prime \prime \prime}$ is obtained from $v$ simply by swapping $a$ and $b$. By Lemma 1 (letting $\{a, b\}$ correspond to $B$ in that lemma), for any alternative $c \notin\{a, b\}$, $P_{f}(V, c)=P_{f}\left(V \cup\left\{v^{\prime \prime \prime}\right\}, c\right)$, and also $P_{f}\left((V-\{v\}) \cup\left\{v^{\prime \prime \prime}\right\}, c\right)=P_{f}\left(V \cup\left\{v^{\prime \prime \prime}\right\}, c\right)$. Now, if we suppose that $P_{f}(V, a)<P_{f}\left(V \cup\left\{v^{\prime \prime \prime}\right\}, a\right)$, then, if the true preferences are given by $V$, the voter corresponding to $v^{\prime}$ would be better off casting $v^{\prime \prime \prime}$ as well (since it will only affect the probabilities of $a$ and $b$ being elected, and $v^{\prime}$ prefers $a$ ). Conversely, if $P_{f}(V, a)>P_{f}\left(V \cup\left\{v^{\prime \prime \prime}\right\}, a\right)$, then the voter corresponding to $v^{\prime \prime}$ would be better off casting $v^{\prime \prime \prime}$ as well. Hence, since $f$ is false-name-proof, $P_{f}(V, a)=P_{f}\left(V \cup\left\{v^{\prime \prime \prime}\right\}, a\right)$. It similarly follows that $P_{f}\left((V-\{v\}) \cup\left\{v^{\prime \prime \prime}\right\}, a\right)=P_{f}\left(V \cup\left\{v^{\prime \prime \prime}\right\}, a\right)$ (since $v^{\prime}$ and $v^{\prime \prime}$ are still present in $\left.(V-\{v\}) \cup\left\{v^{\prime \prime \prime}\right\}\right)$. Hence, $P_{f}(V, a)=P_{f}\left((V-\{v\}) \cup\left\{v^{\prime \prime \prime}\right\}, a\right)$.

Now let us return to the general case where $a$ and $b$ are not necessarily adjacent in $v$ and $v^{\prime \prime \prime}$. Let $v^{\prime \prime \prime \prime}$ be the result of improving $b$ 's position in $v$ to just after $a$, and let $v^{\prime \prime \prime \prime \prime \prime}$ be the result of swapping $a$ and $b$ in $v^{\prime \prime \prime \prime}$. Using Lemma $2, P_{f}(V, a)=P_{f}((V-\{v\}) \cup$ $\left.\left\{v^{\prime \prime \prime \prime}\right\}, a\right)$; using the above argument, $P_{f}\left((V-\{v\}) \cup\left\{v^{\prime \prime \prime \prime}\right\}, a\right)=P_{f}((V-\{v\}) \cup$ $\left.\left\{v^{\prime \prime \prime \prime \prime \prime}\right\}, a\right)$; and using Lemma 2 again, $P_{f}\left((V-\{v\}) \cup\left\{v^{\prime \prime \prime \prime \prime}\right\}, a\right)=P_{f}((V-\{v\}) \cup$ $\left.\left\{v^{\prime \prime \prime}\right\}, a\right)$.

In the next lemma, we use the previous lemmas to reduce a set of votes to a particular pair of votes, without affecting $a$ 's probability of winning.

Lemma 4 Given a nonempty multiset of votes $V$ and a distinguished alternative a, let $B$ be the set of alternatives that are ranked before a by every vote in $V$, let $C$ be the set of alternatives that are ranked before a by some votes in $V$ and after a by others, and let $D$ be the set of alternatives that are ranked after a by every vote in $V$. Let $v$ (not necessarily in $V$ ) be a vote of the form $B \succ a \succ C \cup D$, and let $v^{\prime}$ (not necessarily in $V$ ) be a vote of the form $B \cup C \succ a \succ D$. Then, if $f$ is anonymity-proof, $P_{f}(V, a)=P_{f}\left(\left\{v, v^{\prime}\right\}, a\right)$.

Proof: By Corollary 1 we can add copies of votes without affecting any winning probabilities, hence we can assume without loss of generality that there are at least 3 votes in $V$. First, we use Lemmas 2 and 3 to change an arbitrary one of $V$ 's votes (let us call this vote $v^{\prime \prime}$ ) into $v^{\prime}$, without changing the probability of $a$ winning, as follows. By Lemma 2 , we only need to get this vote in the form $B \cup C \succ a \succ D$. $v^{\prime \prime}$ must already be of the form $B \cup C_{1} \succ\{a\} \succ D \cup C_{2}$ (for some partition $C=C_{1} \cup C_{2}$ ), hence we only need to move each element $c \in C_{2}$ ahead of $a$. We can use Lemma 3 to do so, unless $v^{\prime}$ is the only vote in which $a$ is ranked ahead of $c$. If this is the case, because there are at least 3 
votes, we can use Lemma 3 to first move $c$ after $a$ in one of the other votes, after which we can move $c$ ahead of $a$ in the vote (that was originally) $v^{\prime \prime}$.

Now that $v^{\prime}$ is one of our votes, we change every other vote into $v$ using Lemmas 2 and 3 , as follows. Again, by Lemma 2, we only need to get such a vote into the form $B \succ a \succ C \cup D$, and the vote must already be of the form $B \cup C_{1} \succ\{a\} \succ D \cup C_{2}$. We can use Lemma 3 to move every $c \in C_{1}$ after $a$, because $v^{\prime}$ ranks $c$ ahead of $a$, and at least one other vote ranks $a$ ahead of $c$.

At this point, we have one vote $v^{\prime}$ and multiple votes $v$. But by Corollary 1 we can remove the duplicates of $v$, obtaining $\left\{v, v^{\prime}\right\}$ without having changed $a$ 's probability of winning.

It should be noted that Lemma 4 does not cover the case where $V=\emptyset$; in this case, neutrality demands that an alternative be chosen uniformly at random. The next lemma characterizes the behavior of an anonymity-proof voting rule when only a single vote is cast.

Lemma 5 Let $v=a_{1} \succ \ldots \succ a_{m}$. Let $f$ be anonymity-proof and neutral, and let $p_{f}^{i}=P_{f}\left(\{v\}, a_{i}\right)$. Then, for some constant $0 \leq k_{f} \leq 1, p_{f}^{i}=k_{f} / m+\left(1-k_{f}\right)(m-i)$. $2 /(m(m-1))$. That is, with probability $k_{f}$ the rule chooses an alternative at random, and with probability $1-k_{f}$ it draws a pair of alternatives at random and chooses the preferred one.

Proof: For an arbitrary $1 \leq i \leq m-2$, consider the votes $v^{\prime}=a_{1} \succ \ldots \succ a_{i-1} \succ$ $a_{i+1} \succ a_{i} \succ a_{i+2} \succ a_{i+3} \succ \ldots \succ a_{m}$ and $v^{\prime \prime}=a_{1} \succ \ldots \succ a_{i-1} \succ a_{i} \succ$ $a_{i+2} \succ a_{i+1} \succ a_{i+3} \succ \ldots \succ a_{m}$. Note that $v, v^{\prime}$, and $v^{\prime \prime}$ are all of the form $a_{1} \succ$ $\ldots \succ a_{i-1} \succ\left\{a_{i}, a_{i+1}, a_{i+2}\right\} \succ a_{i+3} \succ \ldots \succ a_{m}$. Thus, by Lemma 1, letting $V=\left\{v, v^{\prime}, v^{\prime \prime}\right\}$, for any $1 \leq j \leq i-1$ or any $i+3 \leq j \leq m, P_{f}\left(V, a_{j}\right)=p_{f}^{j}$. It follows that $P_{f}\left(V, a_{i}\right)+P_{f}\left(V, a_{i+1}\right)+P_{f}\left(V, a_{i+2}\right)=p_{f}^{i}+p_{f}^{i+1}+p_{f}^{i+2}$. Moreover, since $v^{\prime \prime}$ can be obtained from $v$ by swapping $a_{i+1}$ and $a_{i+2}$, by Lemma $1, P_{f}\left(V, a_{i}\right)=$ $P\left(\left\{v, v^{\prime}\right\}, a_{i}\right)$. Again by Lemma 1 , for any $1 \leq j \leq i-1$ or any $i+2 \leq j \leq m$, $P_{f}\left(\left\{v, v^{\prime}\right\}, a_{j}\right)=p_{f}^{j}$. It follows that $P_{f}\left(\left\{v, v^{\prime}\right\}, a_{i}\right)+P_{f}\left(\left\{v, v^{\prime}\right\}, a_{i+1}\right)=p_{f}^{i}+p_{f}^{i+1}$; moreover, by neutrality, $P_{f}\left(\left\{v, v^{\prime}\right\}, a_{i}\right)=P_{f}\left(\left\{v, v^{\prime}\right\}, a_{i+1}\right)=\left(p_{f}^{i}+p_{f}^{i+1}\right) / 2$. Hence $P_{f}\left(V, a_{i}\right)=\left(p_{f}^{i}+p_{f}^{i+1}\right) / 2$. It can symmetrically be shown (using the vote set $\left\{v, v^{\prime \prime}\right\}$ ) that $P_{f}\left(V, a_{i+2}\right)=\left(p_{f}^{i+1}+p_{f}^{i+2}\right) / 2$. It follows that $P_{f}\left(V, a_{i+1}\right)=p_{f}^{i}+p_{f}^{i+1}+p_{f}^{i+2}-$ $\left(p_{f}^{i}+p_{f}^{i+1}\right) / 2-\left(p_{f}^{i+1}+p_{f}^{i+2}\right) / 2=\left(p_{f}^{i}+p_{f}^{i+2}\right) / 2$. However, we can obtain another expression for $P_{f}\left(V, a_{i+1}\right)$ as follows. By Lemma 1, we can add votes $a_{1} \succ \ldots \succ$ $a_{i-1} \succ a_{i+1} \succ a_{i+2} \succ a_{i} \succ a_{i+3} \succ \ldots \succ a_{m}$ and $a_{1} \succ \ldots \succ a_{i-1} \succ a_{i+2} \succ$ $a_{i} \succ a_{i+1} \succ a_{i+3} \succ \ldots \succ a_{m}$ without affecting the probability of $a_{i+1}$ winning. We can also add the vote $a_{1} \succ \ldots \succ a_{i-1} \succ a_{i+2} \succ a_{i+1} \succ a_{i} \succ a_{i+3} \succ \ldots \succ a_{m}$, by adding a copy of $v^{\prime}$ using Corollary 1 and then moving $a_{i+2}$ up two places (which does not affect the probability of $a_{i+1}$ winning, by Lemma 3 ). But in the resulting vote set, $a_{i}, a_{i+1}$, and $a_{i+2}$ are symmetric, and because by Lemma 1 all the other alternatives still have the same probability, it follows that $P_{f}\left(V, a_{i+1}\right)=\left(p_{f}^{i}+p_{f}^{i+1}+p_{f}^{i+2}\right) / 3$. Subtracting the latter expression for $P_{f}\left(V, a_{i+1}\right)$ from the former we obtain $0=\left(p_{f}^{i}+\right.$ $\left.p_{f}^{i+2}\right) / 6-p_{f}^{i+1} / 3$, or equivalently $p_{f}^{i+2}-p_{f}^{i+1}=p_{f}^{i+1}-p_{f}^{i}$. It follows that $p_{f}^{i}$ is a linear function of $i . \sum_{i=1}^{m} p_{f}^{i}=1$, so the average value of $p_{f}^{i}$ must be $1 / m$. Moreover, the $p_{f}^{i}$ must be nonincreasing in $i$ (otherwise, if $v$ is the only vote, that voter would prefer to not participate and obtain the uniform distribution over alternatives). The most 
sharply decreasing function would set $p_{f}^{i}=(m-i) \cdot 2 /(m(m-1))$ so that $p_{f}^{m}=0$ and $p_{f}^{1}=2 / m$. Hence, any correct function for $p_{f}^{i}$ must be a weighted average of this function and the constant function that gives probability $1 / m$ to each alternative.

The final lemma characterizes the probability of $a$ winning in the special pair of votes from Lemma 4, using Lemma 5.

Lemma 6 Let $v$ be a vote of the form $B \succ a \succ C \cup D$, and let $v^{\prime}$ be a vote of the form $B \cup C \succ a \succ D$. Then, if $f$ is anonymity-proof and neutral, $P_{f}\left(\left\{v, v^{\prime}\right\}, a\right)=$ $k_{f} / m+\left(1-k_{f}\right)(2|D|+|C|) /(m(m-1))$, where $k_{f}$ is defined as in Lemma 5. That is, the probability that a wins is the same as under the following rule for selecting the winner: with probability $k_{f}$ the rule chooses an alternative at random; with probability $1-k_{f}$ it draws a pair of alternatives at random, and if every vote prefers the same alternative between the two, it chooses that alternative, otherwise it flips a fair coin to decide between the two alternatives.

Proof: Using Lemma 2, we can assume without loss of generality that $v$ is of the form $B \succ a \succ C \succ D$, that $v^{\prime}$ is of the form $B \succ C \succ a \succ D$, and moreover that the internal orderings of $B$ and $D$ are the same in both votes. By Lemmas 1 and 5, this implies that the alternative ranked $i$ th in $B$ wins with probability $p_{f}^{i}$, and the alternative ranked $i$ th in $D$ wins with probability $p_{f}^{|B|+|C|+1+i}$. Now, suppose that $P_{f}\left(\left\{v, v^{\prime}\right\}, a\right)<$ $k_{f} / m+\left(1-k_{f}\right)(2|D|+|C|) /(m(m-1))$. Let us consider the utility vector $\vec{u}=$ $(1-\epsilon, 1-2 \epsilon, \ldots, 1-(|B|+1) \epsilon,(|C|+|D|) \epsilon,(|C|+|D|-1) \epsilon, \ldots, \epsilon)$. Then, if the true preferences are given by $\left\{v, v^{\prime}\right\}$, the voter casting $v$ has utility vector $\vec{u}$, and $\epsilon$ is sufficiently small, then the voter casting $v$ has an incentive to cast a number of additional votes of the form $B \succ\{a\} \cup C \succ D$ (with $B$ and $D$ in the same order as in $v$ ), in such a way that all alternatives in $\{a\} \cup C$ are symmetric in the resulting vote set. This is because (as $\epsilon \rightarrow 0$ ) she effectively seeks to maximize the probability of one of $B \cup\{a\}$ winning; by Lemma 1 , her additional votes will not affect the winning probabilities of the alternatives in $B$ and $D$; and by neutrality, the resulting probability of $a$ winning will be $(1 /(|C|+$ 1)) $\sum_{i=|B|+1}^{|B|+|C|+1} p_{f}^{i}=(1 /(|C|+1)) \sum_{i=|B|+1}^{|B|+|C|+1} k_{f} / m+\left(1-k_{f}\right)(m-i) \cdot 2 /(m(m-1))=$ $k_{f} / m+\left(1-k_{f}\right)(m-|B|-1+m-|B|-|C|-1) / 2 \cdot 2 /(m(m-1))=k_{f} / m+$ $\left(1-k_{f}\right)(2 m-2|B|-|C|-2) /(m(m-1))=k_{f} / m+\left(1-k_{f}\right)(2|D|+|C|) /(m(m-$ $1))$. Hence, false-name-proofness implies $P_{f}\left(\left\{v, v^{\prime}\right\}, a\right) \geq k_{f} / m+\left(1-k_{f}\right)(2|D|+$ $|C|) /(m(m-1))$. By a symmetric argument, in which the voter corresponding to $v^{\prime}$ effectively tries to minimize the probability of one of $\{a\} \cup D$ winning, it can be shown that $P_{f}\left(\left\{v, v^{\prime}\right\}, a\right) \leq k_{f} / m+\left(1-k_{f}\right)(2|D|+|C|) /(m(m-1))$.

Using the last three lemmas, the main result is now easy to prove:

Theorem 1 The class of voting rules $f$ that are anonymity-proof and neutral consists exactly of the following rules. With some probability $k_{f} \in[0,1]$, we choose an alternative at random. With probability $1-k_{f}$ it draws a pair of alternatives at random. If every vote prefers the same alternative between the two (and there is at least one vote), then it chooses that alternative. Otherwise, it flips a fair coin to decide between the two alternatives. (All these rules are also false-name-proof in a stronger sense where the voter need not cast any vote with her true preferences, and this also implies that they are all strategy-proof.)

Proof: Let us first show that these rules indeed have the desired properties. They are clearly neutral. Conditional on a single random alternative being chosen, voters have 
no incentive to use false names or to not participate. Conditional on a random pair $a, b$ of alternatives being drawn, there are four possibilities for a voter (who, without loss of generality, prefers $a$ ):

1. There are no other votes. In this case, the voter has a strict incentive to participate so that $a$ is chosen, and no incentive to use false names.

2. All other votes prefer $a$. In this case, the voter has no incentive to use false names or not participate, since $a$ will be chosen in any case.

3. All other votes prefer $b$. In this case, the voter has a strict incentive to participate so that at least a coin is flipped, and no incentive to use false names.

4. There are other votes that prefer $a$ and other votes that prefer $b$. In this case, the voter has no incentive to use false names or not participate, since a coin will be flipped in any case.

We now show that there are no other rules with the desired properties. Let $f$ be anonymity-proof and neutral. Lemma 5 defines $k_{f}$ for this rule. Now, for an arbitrary multiset of votes $V$ and an arbitrary alternative $a$, Lemma 4 shows how to convert $V$ to a particular set of two votes $\left\{v, v^{\prime}\right\}$, in a way that preserves $a$ 's probability of winning, and also preserves $a$ 's relationship to any other alternative $b$ in the following sense:

- If all votes prefer $a$ to $b$ in $V$, the same is true in $\left\{v, v^{\prime}\right\}$.

- If all votes prefer $b$ to $a$ in $V$, the same is true in $\left\{v, v^{\prime}\right\}$.

- If some but not all votes prefer $a$ to $b$ in $V$, the same is true in $\left\{v, v^{\prime}\right\}$.

Finally, Lemma 6 shows that for this set of two votes $\left\{v, v^{\prime}\right\}$, alternative's $a$ 's probability of winning is as in the claim of this theorem. Because of the preservation properties of the conversion, this must also be true for the original set of votes $V$.

\section{Discussion}

In this section, we study some corollaries of the main result, and make some comparisons to rules that are only strategy-proof.

The characterization makes it clear that the optimal anonymity-proof rule (in any reasonable sense of the word "optimal") is the one corresponding to $k_{f}=0$, since this rule maximizes the probability that we can at least choose the better of two alternatives (if all votes agree). Even this rule is limited in the extent to which it can respond to the votes:

Corollary 2 Under an anonymity-proof rule, the probability of any given alternative a winning is at most $2 / \mathrm{m}$ (for any multiset of votes). This probability is attained if and only if $k_{f}=0$ and all votes rank a first.

This is in sharp contrast to the class of strategy-proof rules. For example, it is strategyproof to draw one of the votes at random and choose its most-preferred alternative (often referred to as the "random-dictator" rule). Under this rule, if an alternative ranks first in all votes, it will be chosen with probability 1 . Also, within the class of strategy-proof rules, there is no rule that is clearly optimal. For example, it is also strategy-proof to 
draw a pair of alternatives at random, and choose the one that is preferred by more voters. Unlike the random-dictator rule, if there is an alternative that ranks first in all votes, this rule does not necessarily choose it; on the other hand, unlike the random-dictator rule, this rule does not run the risk of choosing an alternative that is ranked last by almost every vote (but first by a few).

Another sharp contrast between strategy-proof rules such as the above two and any anonymity-proof rule is the following. For the winning alternative not to be chosen uniformly at random, anonymity-proof rules require complete agreement on at least one pair of alternatives:

Corollary 3 If $V$ and $a$ are such that for any $b \neq a$, there is $a$ vote in $V$ that prefers a to $b$, as well as one that prefers $b$ to a, then for any anonymity-proof voting rule, $P_{f}(V, a)=$ $1 / m$.

Proof: If a pair of alternatives including $a$ is drawn, a coin toss will result. Therefore $P_{f}(V, a)=k_{f} / m+\left(1-k_{f}\right)(2 / m)(1 / 2)=1 / m$.

Our final corollary is computational in nature:

Corollary 4 For any anonymity-proof voting rule $f$, it is easy to compute $P_{f}(V, a)$ for any inputs $V$ and $a$.

Proof: If $m_{1}$ is the number of alternatives that are always ranked after $a$, and $m_{2}$ is the number of alternatives that are sometimes ranked before $a$, and sometimes ranked after $a$, then $P_{f}(V, a)=k_{f} / m+\left(1-k_{f}\right)\left(2 m_{1}+m_{2}\right) /(m(m-1))$.

It is not immediately clear if a similar result holds for strategy-proof rules. For one, it is not immediately clear if there is a natural concise representation for such rules. (In contrast, per Theorem 1, an anonymity-proof neutral voting rule can be represented by a single number $k_{f} \in[0,1]$.)

\section{Future research}

Although Theorem 1 completely characterizes anonymity-proof neutral voting rules, much remains to be done in future research. The most natural next direction to take is to consider settings where the space of possible preferences is restricted. It is well-known that such restrictions can introduce very satisfactory strategy-proof rules. For example, in many settings there is a natural order on the alternatives (e.g. in political elections, we can order candidates by how far to the left of the political spectrum they are). In such a setting, a voter's preferences are said to be single-peaked if she always prefers alternatives that are closer to her most-preferred alternative to alternatives that are further away (when these alternatives are on the same side of the most-preferred alternative) [11]. It is wellknown that when preferences are single-peaked, choosing the most preferred alternative of the median voter (the voter that, if we sort the voters by their most preferred alternatives, ends up in the middle) is strategy-proof, and (if the number of voters is odd) this alternative will be preferred to any other alternative by more than half of the voters (i.e. it is the Condorcet winner). Single-peakedness can only be of limited help for anonymityproofness: for example, when there are only two alternatives, single-peakedness does not restrict preferences at all, so we cannot do anything more than in the general case. However, there may be other restrictions that are more fruitful. Specific application settings 
can also allow for more positive results, as has already been shown to be the case for combinatorial auctions. (In a sense, such settings correspond to a very special way of restricting preferences.) Finally, if no good anonymity-proof mechanisms turn out to exist for a setting that we are interested in, then we need to consider other options. One natural solution is to verify agents' identities, that is, to check whether multiple preference reports came from the same agent. It is generally not necessary to verify the identities of all agents; rather, it suffices to verify those of a select few based on the submitted preference reports [17]. A final approach is to try to stop the problem at the source and make it impossible or impractical for an agent to sign up for more than one account, but it seems difficult to do so without compromising the anonymity of the Internet.

\section{References}

[1] Nir Ailon. Aggregation of partial rankings, p-ratings and top-m lists. In Proceedings of the Annual ACM-SIAM Symposium on Discrete Algorithms (SODA), 2007.

[2] Nir Ailon, Moses Charikar, and Alantha Newman. Aggregating inconsistent information: Ranking and clustering. In Proceedings of the Annual Symposium on Theory of Computing (STOC), 2005.

[3] Aaron Archer, Christos Papadimitriou, Kunal Talwar, and Eva Tardos. An approximate truthful mechanism for combinatorial auctions with single parameter agents. In Proceedings of the Annual ACM-SIAM Symposium on Discrete Algorithms (SODA), 2003.

[4] Moshe Babaioff, Nicole Immorlica, and Robert Kleinberg. Matroids, secretary problems, and online mechanisms. In Proceedings of the Annual ACM-SIAM Symposium on Discrete Algorithms (SODA), 2007.

[5] Maria-Florina Balcan, Avrim Blum, Jason D. Hartline, and Yishay Mansour. Mechanism design via machine learning. In Proceedings of the Annual Symposium on Foundations of Computer Science (FOCS), pages 605-614, 2005.

[6] John Bartholdi, III and James Orlin. Single transferable vote resists strategic voting. Social Choice and Welfare, 8(4):341-354, 1991.

[7] John Bartholdi, III, Craig Tovey, and Michael Trick. The computational difficulty of manipulating an election. Social Choice and Welfare, 6(3):227-241, 1989.

[8] John Bartholdi, III, Craig Tovey, and Michael Trick. Voting schemes for which it can be difficult to tell who won the election. Social Choice and Welfare, 6:157-165, 1989.

[9] John Bartholdi, III, Craig Tovey, and Michael Trick. How hard is it to control an election? Math. Comput. Modelling, 16(8-9):27-40, 1992. Formal theories of politics, II.

[10] Sushil Bikhchandani, Shurojit Chatterji, Ron Lavi, Ahuva Mu'alem, Noam Nisan, and Arunava Sen. Weak monotonicity characterizes deterministic dominant strategy implementation. Econometrica, 74(4):1109-1132, 2006.

[11] Duncan Black. On the rationale of group decision-making. Journal of Political Economy, 56(1):23-34, 1948. 
[12] Avrim Blum, Tuomas Sandholm, and Martin Zinkevich. Online algorithms for market clearing. Journal of the ACM, 53(5):845-879, 2006.

[13] Felix Brandt, Felix Fischer, and Paul Harrenstein. The computational complexity of choice sets. In Theoretical Aspects of Rationality and Knowledge (TARK), Brussels, Belgium, 2007.

[14] Ed H. Clarke. Multipart pricing of public goods. Public Choice, 11:17-33, 1971.

[15] William Cohen, Robert Schapire, and Yoram Singer. Learning to order things. Journal of Artificial Intelligence Research, 10:213-270, 1999.

[16] Vincent Conitzer. Computing Slater rankings using similarities among candidates. In Proceedings of the National Conference on Artificial Intelligence (AAAI), Boston, MA, 2006.

[17] Vincent Conitzer. Limited verification of identities to induce false-name-proofness. In Theoretical Aspects of Rationality and Knowledge (TARK), Brussels, Belgium, 2007.

[18] Vincent Conitzer, Andrew Davenport, and Jayant Kalagnanam. Improved bounds for computing Kemeny rankings. In Proceedings of the National Conference on Artificial Intelligence (AAAI), Boston, MA, 2006.

[19] Vincent Conitzer and Tuomas Sandholm. Universal voting protocol tweaks to make manipulation hard. In Proceedings of the Eighteenth International Joint Conference on Artificial Intelligence (IJCAI), pages 781-788, Acapulco, Mexico, 2003.

[20] Vincent Conitzer and Tuomas Sandholm. Self-interested automated mechanism design and implications for optimal combinatorial auctions. In Proceedings of the ACM Conference on Electronic Commerce (EC), pages 132-141, New York, NY, USA, 2004.

[21] Vincent Conitzer and Tuomas Sandholm. Communication complexity of common voting rules. In Proceedings of the ACM Conference on Electronic Commerce (EC), pages 78-87, Vancouver, Canada, 2005.

[22] Vincent Conitzer and Tuomas Sandholm. Nonexistence of voting rules that are usually hard to manipulate. In Proceedings of the National Conference on Artificial Intelligence (AAAI), Boston, MA, 2006.

[23] Vincent Conitzer, Tuomas Sandholm, and Jérôme Lang. When are elections with few candidates hard to manipulate? Journal of the ACM, 2007. To appear.

[24] Don Coppersmith, Lisa Fleischer, and Atri Rudra. Ordering by weighted number of wins gives a good ranking for weighted tournaments. In Proceedings of the Annual ACM-SIAM Symposium on Discrete Algorithms (SODA), 2006.

[25] Andrew Davenport and Jayant Kalagnanam. A computational study of the Kemeny rule for preference aggregation. In Proceedings of the National Conference on Artificial Intelligence (AAAI), pages 697-702, San Jose, CA, 2004.

[26] Cynthia Dwork, Ravi Kumar, Moni Naor, and D. Sivakumar. Rank aggregation methods for the web. In Proceedings of the 10th World Wide Web Conference, pages 613-622, 2001. 
[27] Edith Elkind and Helger Lipmaa. Hybrid voting protocols and hardness of manipulation. In Annual International Symposium on Algorithms and Computation (ISAAC), 2005.

[28] Piotr Faliszewski, Edith Hemaspaandra, and Lane A. Hemaspaandra. The complexity of bribery in elections. In Proceedings of the National Conference on Artificial Intelligence (AAAI), Boston, MA, 2006.

[29] Allan Gibbard. Manipulation of voting schemes: a general result. Econometrica, 41:587-602, 1973.

[30] Allan Gibbard. Manipulation of schemes that mix voting with chance. Econometrica, 45:665-681, 1977.

[31] Jerry Green and Jean-Jacques Laffont. Characterization of satisfactory mechanisms for the revelation of preferences for public goods. Econometrica, 45:427-438, 1977.

[32] Theodore Groves. Incentives in teams. Econometrica, 41:617-631, 1973.

[33] Mohammad T. Hajiaghayi, Robert Kleinberg, Mohammad Mahdian, and David C. Parkes. Online auctions with re-usable goods. In Proceedings of the ACM Conference on Electronic Commerce (EC), pages 165-174, Vancouver, Canada, 2005.

[34] Mohammad T. Hajiaghayi, Robert Kleinberg, and David C. Parkes. Adaptive limited-supply online auctions. In Proceedings of the ACM Conference on Electronic Commerce (EC), pages 71-80, New York, NY, USA, 2004.

[35] Edith Hemaspaandra and Lane A. Hemaspaandra. Dichotomy for voting systems. Journal of Computer and System Sciences, 73(1):73-83, 2007.

[36] Edith Hemaspaandra, Lane A. Hemaspaandra, and Joerg Rothe. Exact analysis of Dodgson elections: Lewis Carroll's 1876 voting system is complete for parallel access to NP. Journal of the ACM, 44(6):806-825, 1997.

[37] Edith Hemaspaandra, Lane A. Hemaspaandra, and Joerg Rothe. Anyone but him: The complexity of precluding an alternative. In Proceedings of the National Conference on Artificial Intelligence (AAAI), Pittsburgh, PA, 2005.

[38] Jérôme Lang. Vote and aggregation in combinatorial domains with structured preferences. In Proceedings of the Twentieth International Joint Conference on Artificial Intelligence (IJCAI), pages 1366-1371, Hyderabad, India, 2007.

[39] Ron Lavi, Ahuva Mu'alem, and Noam Nisan. Towards a characterization of truthful combinatorial auctions. In Proceedings of the Annual Symposium on Foundations of Computer Science (FOCS), pages 574-583, 2003.

[40] Ron Lavi and Chaitanya Swamy. Truthful and near-optimal mechanism design via linear programming. In Proceedings of the Annual Symposium on Foundations of Computer Science (FOCS), pages 595-604, 2005.

[41] Daniel Lehmann, Lidian Ita O'Callaghan, and Yoav Shoham. Truth revelation in rapid, approximately efficient combinatorial auctions. Journal of the ACM, 49(5):577-602, 2002. 
[42] Aranyak Mehta, Tim Roughgarden, and Mukund Sundararajan. Beyond Moulin mechanisms. In Proceedings of the ACM Conference on Electronic Commerce (EC), San Diego, CA, USA, 2007.

[43] Roger Myerson. Incentive compatibility and the bargaining problem. Econometrica, 41(1), 1979.

[44] Roger Myerson. Optimal auction design. Mathematics of Operations Research, 6:58-73, 1981.

[45] Noam Nisan and Amir Ronen. Algorithmic mechanism design. Games and Economic Behavior, 35:166-196, 2001.

[46] Maria Silvia Pini, Francesca Rossi, Kristen Brent Venable, and Toby Walsh. Aggregating partially ordered preferences: possibility and impossibility results. In Theoretical Aspects of Rationality and Knowledge (TARK), Singapore, 2005.

[47] Maria Silvia Pini, Francesca Rossi, Kristen Brent Venable, and Toby Walsh. Incompleteness and incomparability in preference aggregation. In Proceedings of the Twentieth International Joint Conference on Artificial Intelligence (IJCAI), Hyderabad, India, 2007.

[48] Ariel D. Procaccia and Jeffrey S. Rosenschein. Average-case tractability of manipulation in voting via the fraction of manipulators. In International Conference on Autonomous Agents and Multi-Agent Systems (AAMAS), Honolulu, HI, USA, 2007.

[49] Ariel D. Procaccia and Jeffrey S. Rosenschein. Junta distributions and the averagecase complexity of manipulating elections. Journal of Artificial Intelligence Research, 28:157-181, February 2007.

[50] Ariel D. Procaccia, Jeffrey S. Rosenschein, and Aviv Zohar. Multi-winner elections: Complexity of manipulation, control and winner-determination. In Proceedings of the Twentieth International Joint Conference on Artificial Intelligence (IJCAI), Hyderabad, India, 2007.

[51] Francesca Rossi, Maria Silvia Pini, Kristen Brent Venable, and Toby Walsh. Strategic voting when aggregating partially ordered preferences. In International Conference on Autonomous Agents and Multi-Agent Systems (AAMAS), pages 685-687, Hakodate, Japan, 2006.

[52] Joerg Rothe, Holger Spakowski, and Joerg Vogel. Exact complexity of the winner problem for Young elections. In Theory of Computing Systems, volume 36(4), pages 375-386. Springer-Verlag, 2003.

[53] Mark Satterthwaite. Strategy-proofness and Arrow's conditions: Existence and correspondence theorems for voting procedures and social welfare functions. Journal of Economic Theory, 10:187-217, 1975.

[54] William Vickrey. Counterspeculation, auctions, and competitive sealed tenders. Journal of Finance, 16:8-37, 1961.

[55] Lirong Xia, Jérôme Lang, and Mingsheng Ying. Sequential voting rules and multiple elections paradoxes. In Theoretical Aspects of Rationality and Knowledge (TARK), Brussels, Belgium, 2007. 
[56] Makoto Yokoo. The characterization of strategy/false-name proof combinatorial auction protocols: Price-oriented, rationing-free protocol. In Proceedings of the Eighteenth International Joint Conference on Artificial Intelligence (IJCAI), pages 733-742, Acapulco, Mexico, 2003.

[57] Makoto Yokoo, Toshihiro Matsutani, and Atsushi Iwasaki. False-name-proof combinatorial auction protocol: Groves mechanism with submodular approximation. In International Conference on Autonomous Agents and Multi-Agent Systems (AA$M A S)$, pages 1135-1142, Hakodate, Japan, 2006.

[58] Makoto Yokoo, Yuko Sakurai, and Shigeo Matsubara. Robust combinatorial auction protocol against false-name bids. Artificial Intelligence, 130(2):167-181, 2001.

[59] Makoto Yokoo, Yuko Sakurai, and Shigeo Matsubara. The effect of false-name bids in combinatorial auctions: New fraud in Internet auctions. Games and Economic Behavior, 46(1):174-188, 2004. 